This is the final peer-reviewed accepted manuscript of:

[Chiral capillary zone electrophoresis in enantioseparation and analysis of cinacalcet impurities: Use of Quality by Design principles in method development, Benedetta Pasquini, Serena Orlandini, Mercedes Villar-Navarro, Claudia Caprini, Massimo Del Bubba, Michal Douša, Alessandro Giuffrida, Roberto Gotti, Sandra Furlanetto, Journal of Chromatography A, 1568 (2018) 205-213]

The final published version is available online at: [10.1016/i.chroma.2018.07.021]

Rights / License:

The terms and conditions for the reuse of this version of the manuscript are specified in the publishing policy. For all terms of use and more information see the publisher's website.

This item was downloaded from IRIS Università di Bologna (https://cris.unibo.it/)

When citing, please refer to the published version. 


\title{
Chiral capillary zone electrophoresis in enantioseparation and analysis of cinacalcet impurities: Use of Quality by Design principles in method development
}

\author{
Benedetta Pasquini ${ }^{a}$, Serena Orlandini ${ }^{\mathrm{a}, *}$, Mercedes Villar-Navarro ${ }^{\mathrm{b}}$, Claudia Caprini ${ }^{\mathrm{a}}$, \\ Massimo Del Bubba ${ }^{\mathrm{a}}$, Michal Douša ${ }^{\mathrm{c}}$, Alessandro Giuffrida ${ }^{\mathrm{d}}$, Roberto Gotti ${ }^{\mathrm{e}}$, \\ Sandra Furlanetto ${ }^{\mathrm{a}, *}$ \\ a Department of Chemistry “U. Schiff”, University of Florence, Via U. Schiff 6, Via della Lastruccia 3-13, 50019, Sesto Fiorentino, Florence, Italy \\ ${ }^{\mathrm{b}}$ Department of Analytical Chemistry, Faculty of Chemistry, University of Seville, 41012, Seville, Spain \\ ' Zentiva, K.S. Praha, a Sanofi Company, U Kabelovny 130, 102 37, Praha 10, Czech Republic \\ ${ }^{\mathrm{d}}$ Department of Chemical Sciences, University of Catania, Viale A. Doria 6, 95125, Catania, Italy \\ e Department of Pharmacy and Biotechnology, University of Bologna, Via Belmeloro 6, 40126, Bologna, Italy
}

\section{A R T I C L E I N F O}

\section{Article history:}

Received 23 March 2018

Received in revised form 29 June 2018

Accepted 4 July 2018

Available online $\mathrm{xxx}$

\section{Keywords:}

Capillary electrophoresis

Chiral separation

Cinacalcet

Impurities

Method operable design region

Quality by Design

\begin{abstract}
A B S T R A C T
A capillary electrophoresis method for the simultaneous determination of the enantiomeric purity and of impurities of the chiral calcimimetic drug cinacalcet hydrochloride has been developed following Quality by Design principles. The scouting phase was aimed to select the separation operative mode and to identify a suitable chiral selector. Among the tested cyclodextrins, (2-carboxyethyl)- $\beta$-cyclodextrin and (2-hydroxypropyl)- $\gamma$-cyclodextrin (HP $\gamma$ CyD) showed good chiral resolving capabilities. The selected separation system was solvent-modified capillary zone electrophoresis with the addition of HP $\gamma C y D$ and methanol. Voltage, buffer $\mathrm{pH}$, methanol concentration and $\mathrm{HP} \gamma \mathrm{CyD}$ concentration were investigated as critical method parameters by a multivariate strategy. Critical method attributes were represented by enantioresolution and analysis time. A Box-Behnken Design allowed the contour plots to be drawn and quadratic and interaction effects to be highlighted. The Method Operable Design Region (MODR) was identified by applying Monte-Carlo simulations and corresponded to the multidimensional zone where both the critical method attributes fulfilled the requirements with a desired probability $\pi \geq 90 \%$. The working conditions, with the MODR limits, corresponded to the following: capillary length, $48.5 \mathrm{~cm}$; temperature, $18^{\circ} \mathrm{C}$; voltage, $26 \mathrm{kV}(26-27 \mathrm{kV})$; background electrolyte, $150 \mathrm{mM}$ phosphate buffer $\mathrm{pH}$ 2.70 (2.60-2.80), $3.1 \mathrm{mM}$ (3.0-3.5 mM) HP $\gamma C y D ; 2.00 \%$ (0.00-8.40\%) v/v methanol. Robustness testing was carried out by a Plackett-Burman matrix and finally a method control strategy was defined. The complete separation of the analytes was obtained in about $10 \mathrm{~min}$. The method was validated following the International Council for Harmonisation guidelines and was applied for the analysis of a real sample of cinacalcet hydrochloride tablets.
\end{abstract}

(c) 2018 Elsevier B.V. All rights reserved.

\section{Introduction}

Cinacalcet hydrochloride (CIN) is the first agent of a new therapeutic class of calcimimetic compounds, which act by increasing the sensitivity of calcium-sensing receptors to the extracellular calcium ions, thus lowering the release of parathyroid hormone [1]. CIN is an approved medication for the treatment of secondary

\footnotetext{
* Corresponding authors.

E-mail addresses: serena.orlandini@unifi.it (S. Orlandini), sandra.furlanetto@unifi.it (S. Furlanetto).
}

hyperparathyroidism in adult patients with chronic kidney disease on dialysis and hypercalcemia in patients with parathyroid carcinoma [2]. It has been commercialized as single $R$ enantiomer, due to the fact that $R$-CIN is about 75 times more potent than its corresponding $S$-enantiomer [3]. According to the drug product producer (Zentiva, Praha), the main potential impurities of CIN which may be found in CIN bulk samples and dosage forms are the distomer $S$-cinacalcet $\left(S\right.$-CIN), impurity $1\left(\mathrm{I}_{1}\right)$ and impurity $2\left(\mathrm{I}_{2}\right)$, whose chemical structures are shown in Fig. 1.

The effective and safe therapy with chiral drugs basically depends on the quality of the pharmaceutical dosage forms, which must be controlled both in terms of drug and potential impuri- 
<smiles>C[C@H](NCCCc1cccc(C(F)(F)F)c1)c1cccc2ccccc12</smiles>

Cinacalcet hydrochloride (CIN)

(R)-N-[1-(1-naphthyl)ethyl]-3-[3-(trifluoromethyl) phenyl]propan-1-amine hydrochloride CAS 364782-34-3

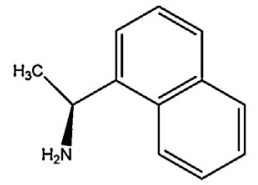

Impurity $1\left(\mathrm{I}_{1}\right)$

$(R)-(+)-1-(1-$ naphthyl)ethylamine CAS 3886-70-2<smiles>C[C@H](NCCCc1cccc(C(F)(F)F)c1)c1cccc2ccccc12</smiles>

$S$-cinacalcet $(S$-CIN)

(S)-N-[1-(1-naphthyl)ethyl]-3-[3-(trifluoromethyl)phenyl] propan-1-amine hydrochloride CAS 694495-47-1<smiles>Cc1cccc(CCCN[C@H](C)c2cccc3ccccc23)c1</smiles>

Impurity $2\left(\mathrm{I}_{2}\right)$

$N$-[3-(3-methylphenyl)propyl] $-N-[(1 R) 1-(1-$ naphthyl) ethyl]amine hydrochloride

CAS 253337-60-9

Fig. 1. Molecular structures of the compounds.

ties content and enantiomeric purity. In particular, the assessment of enantiomeric purity is required by regulatory agencies prior to the marketing of optically active drugs, and in this context the efficient separation of enantiomers represents a fundamental analytical task. Enantioseparation can be effectively obtained with several chromatographic techniques including HPLC, ultra-high performance liquid chromatography, nano-liquid chromatography and TLC [4-7]. Anyway, for routine analysis it is important to have at disposal fast and low cost analytical methods with simple sample preparation and eco-friendly characteristics as small reagent consumption. CE easily fulfills these requirements and has been effectively applied during all stages of drug discovery as well as for the quality control of the finished pharmaceutical products. Among the well-known advantages of CE there are its inherent flexibility as well as the various available operative modes, high separation efficiency, low sample and reagent consumption [8]. In pharmaceutical analysis, CE has been widely applied to the determination of the main component as well as for the purity of drugs with regard to related substances and stereoisomeric impurities [9]. It represents one of the major techniques not only for the achievement of analytical scale enantioseparations but also for a better understanding of the fine intermolecular recognition mechanisms which take place [10-13]. In addition, CE utilizes a wide spectrum of chiral selectors as buffer additives for chiral separation, without the need for expensive columns as for chromatography. The most commonly used chiral selectors are cyclodextrins (CyDs), due to their wide variety of cavity size, side chain, degree of substitution and charge [14]. When added to plain buffers, CyDs are able to resolve enantiomeric guests due to formation of diastereomeric inclusion complexes, which present different electrophoretic mobilities. When the background electrolyte (BGE) contains pseudostationary phases as micelles or microemulsions, it has been recently suggested that more complex phenomena may take place $[15,16]$. In the latter case also the monomers of surfactant can interact with the CyD, possibly involving the formation of ternary complexes $1: 1: 1$ of CyD:enantiomer:surfactant, or giving rise to the displacement of CyD bound enantiomeric guests with surfactant tails due to competitive binding. In any case the affinity of the enantiomers for the CyD can be effectively modulated.

The analysis of CIN and two process-related impurities has been performed by HPLC [17], and stability indicating chromatographic methods involving forced degradation studies have been recently presented [18-21]. Enantioseparation of CIN and its S- enantiomer was obtained by chiral normal phase HPLC [22], by indirect reversed-phase HPLC with chiral auxiliaries as derivatizing agents and by direct thin-layer chromatography [23], and by polysaccharide chiral reversed-phase HPLC [24]. Recently, a LC/MSMS method for separation and determination of CIN enantiomers in rat plasma on chirobiotic $\mathrm{V}$ column packed with vancomycin has been presented [25]. To the best of our knowledge, CE methods for the determination of $I_{1}$ and $I_{2}$ have not been reported in previous literature. There is only one reported CE method for the chiral separation of CIN [26], which was developed by univariate experiments to test the enantiopurity of CIN in tablets without considering any of the other potential impurities which can be found in the pharmaceutical dosage form.

For the first time in the literature, the purpose of this study was to set up a CE method for the simultaneous determination of CIN, its chiral impurity S-CIN and its main impurities $\mathrm{I}_{1}$ and $\mathrm{I}_{2}$ in pharmaceutical formulations, for fulfilling the requirements of an effective Quality Control strategy. Method development has been carried out by Quality by Design (QbD), a risk-management approach following the recent guidelines in the pharmaceutical field [27]. QbD has been recently focused in the field of separation methods $[28,29]$, and its application in this study made it possible to identify not only a single optimum point but a multidimensional zone where the desired quality of analytical data for determining all the four analytes was achieved.

QbD has been defined as "a systematic approach to development that begins with predefined objectives and emphasizes product and process understanding and process control, based on sound science and quality risk management" and has the aim of improving product quality and of increasing regulatory flexibility [27]. In terms of application to analytical methods, Analytical Quality by Design (AQbD) emphasizes the need to thoroughly understand the analytical system by an in-depth study of critical method parameters (CMPs) based on risk assessment and multivariate tools [28,29]. Such strategy enables the robust optimization of the analytical method in compliance with the recommendations of US Food and Drug Administration (US FDA) [30] and with the guidelines of International Council for Harmonisation (ICH) [27], and represents a great step forward with respect to the traditional quality-bytesting approach. As a matter of facts, the latter furnishes data and information limited to the experiments run by the operator, without studying the possible interactions between CMPs and without building a model relating the CMPs to critical method attributes 
(CMAs). On the other hand, by AQbD it is possible to properly manage the risk and to identify a probabilistic design space, which in terms of analytical concept should be better defined as the method operable design region (MODR) [31]. The MODR corresponds to a set of experimental conditions where the desired quality, measured by CMAs values, is achieved with a selected probability. Thus, flexibility is augmented and possible method modifications which could be necessary in the future are facilitated. This is possible thanks to the knowledge of their potential impact on the method performances, gained by the risk-based approach.

The pharmaceutical regulatory requirements and the advantages of QbD methodology have led to an increase in the use of QbD compliant analytical methods for the determination of impurities through the use of experimental design and the computation of a MODR. The use of experimental design allows the exploration of the effects and of the interactions of the CMPs by the calculation of polynomial models relating the CMPs to the CMAs. On the other hand, the MODR is suitable to predict optimal analytical conditions within the experimental domain, with the associated probability of fulfilling the CMAs requirements. Recent examples of applications of AQbD for the analysis of impurities mainly concern the development of separation methods by HPLC [32-34], supercritical fluid chromatography [35] and CE in its various operative modes [36-38], with the latter technique also effectively used for the determination of enantiomeric impurities [39,40].

In this study, QbD scouting allowed capillary zone electrophoresis with the addition of methanol ( $\mathrm{MeOH}$ ) and (2-hydroxypropyl)$\gamma$-cyclodextrin (HP $\gamma \mathrm{CyD}$ ) to be selected as separation system. Response Surface Methodology (RSM) [41] was carried out by using a Box-Behnken design for investigating the effects of the CMPs (voltage, buffer $\mathrm{pH}, \mathrm{MeOH}$ concentration and $\mathrm{HP} \gamma \mathrm{CyD}$ concentration) on the CMAs, represented by enantioresolution and analysis time. The calculated models and Monte-Carlo simulations [42] led to the definition of the MODR. Validation of the method was carried out according to ICH guidelines [43], and the method was applied for the analysis of a real sample of CIN tablets.

\section{Materials and methods}

\subsection{Chemicals and reagents}

Reference standards of CIN and its impurities (S-CIN, $\mathrm{I}_{1}, \mathrm{I}_{2}$ ) were kindly supplied by Zentiva (Praha, Czech Republic), as well as Mimpara ${ }^{\circledR}$ coated tablets labeled to contain $90 \mathrm{mg}$ of CIN and coated tablets excipients, e.g. pre-gelatinised starch, microcrystalline cellulose, povidone, crospovidone, magnesium stearate, colloidal anhydrous silica, lactose monohydrate, hypromellose, titanium dioxide (E171), glycerol triacetate, FD\&C Blue (E132), yellow iron oxide (E172).

Boric acid, 86.1\% phosphoric acid, acetic acid, $\mathrm{MeOH}$ (HPLC grade), metformin hydrochloride (MET), n-butanol, ethanol, acetonitrile, urea, all the CyDs tested with their degree of substitution (D.S.) in brackets, i.e. $\alpha$-cyclodextrin, $\gamma$-cyclodextrin, methyl- $\beta$-cyclodextrin (D.S. 1.5-2.1), heptakis (2,6-di-O-methyl- $\beta$-cyclodextrin) (D.S. 7.0), heptakis (2,3,6-tri-O-methyl- $\beta$-cyclodextrin) (D.S. 3.0), (2-hydroxyethyl)$\beta$-cyclodextrin (D.S. 0.7), (2-hydroxypropyl)- $\alpha$-cyclodextrin (D.S. 0.6), (2-hydroxypropyl)- $\beta$-cyclodextrin (D.S. 0.6), HP $\gamma$ CyD (D.S. 0.6), sulfated- $\beta$-cyclodextrin sodium salt (D.S. 12-15), (2carboxyethyl)- $\beta$-cyclodextrin sodium salt (CE $\beta$ CyD) (D.S. 3.0) and all the other chemicals used were from Sigma-Aldrich (St. Louis, MO, USA). Water used for the preparation of the solutions and running buffers was purified by Elix and Simplicity 185 systems (Millipore, Billerica, MA, USA).

\subsection{Solutions and sample preparation}

Standard stock solutions of CIN $\left(10 \mathrm{mg} \mathrm{ml}^{-1}\right)$, of the impurities $\left(1 \mathrm{mg} \mathrm{ml}^{-1}\right)$ and of the internal standard $\operatorname{MET}\left(1 \mathrm{mg} \mathrm{ml}^{-1}\right)$ were prepared in ethanol and stored at $4{ }^{\circ} \mathrm{C}$ for a week. Working standard solutions were daily prepared in water by adequate dilution. Running buffer solutions were prepared by adjusting the $\mathrm{pH}$ value of a proper volume of the corresponding $0.5 \mathrm{M}$ acid or mixture of acids by $\mathrm{NaOH}$ and by dilution up to the desired concentration. Britton-Robinson buffers were prepared from a mixture of acetic acid, phosphoric acid and boric acid. Additives (CyDs, organic solvents) were directly added to the plain buffers in order to obtain the final BGE. For sample preparation, twenty Mimpara ${ }^{\circledR}$ tablets were weighed, crushed and powdered. The equivalent of $100 \mathrm{mg}$ CIN was accurately weighed and transferred to a $10 \mathrm{~mL}$ beaker and dissolved in ethanol. The suspension was stirred and sonicated for $10 \mathrm{~min}$. One milliliter of the mixture was centrifuged and $50 \mu \mathrm{L}$ of the surnatant was diluted in a vial up to $500 \mu \mathrm{L}$ by addition of $30 \mu \mathrm{L}$ of MET stock solution and $420 \mu \mathrm{L}$ of water. The final test concentration of CIN was about $1 \mathrm{mg} \mathrm{mL}^{-1}$ and MET concentration was $0.06 \mathrm{mg} \mathrm{mL}^{-1}$.

\subsection{Capillary electrophoresis apparatus and analyses}

The CE experiments were performed on a $\mathrm{HP}{ }^{3 \mathrm{D}} \mathrm{CE}$ system (Agilent Technologies, Waldbronn, Germany) equipped with an on-column UV-vis DAD and an air thermostating system. The CE instrument was driven and the data were collected by the software ${ }^{3 \mathrm{D}}$ CE ChemStation Rev.09.01 (Agilent Technologies). Uncoated fused silica capillaries ( $50 \mu \mathrm{m}$ inner diameter, $365 \mu \mathrm{m}$ outer diameter, total length $48.5 \mathrm{~cm}$, effective length $40.0 \mathrm{~cm}$ ) were used for running the analyses and were purchased from Unifibre (Settimo Milanese, Italy). The detection wavelength was $220 \mathrm{~nm}$ and the samples were hydrodynamically injected ( 50 mbar for $3 \mathrm{~s}$ ), followed by a BGE plug at $50 \mathrm{mbar}$ for $3 \mathrm{~s}$. Each new capillary was flushed with $1 \mathrm{M} \mathrm{NaOH}, 0.1 \mathrm{M} \mathrm{NaOH}$ and water (5 min each). Between injections, the capillary was washed with methanol, $0.1 \mathrm{M}$ $\mathrm{NaOH}$, water for $1 \mathrm{~min}$ each, and then with the proper BGE for $3 \mathrm{~min}$. The working conditions (with the interval corresponding to the MODR) were: temperature, $18^{\circ} \mathrm{C}$; voltage, $26 \mathrm{kV}(26-27 \mathrm{kV})$; BGE, 150 mM BGE phosphate buffer, pH 2.70 (2.60-2.80), $3.1 \mathrm{mM}$ (3.0-3.5 mM) HPyCyD concentration, 2.00\% (0.00-8.40\%) v/v $\mathrm{MeOH}$ concentration.

\subsection{Calculations and softwares}

In order to obtain the calibration graphs, the peak corrected area (area/migration time) ratios were plotted against the corresponding analyte concentration. Two samples for each of five different concentration values of the compounds were analyzed with the concentration of MET (internal standard) set at $0.06 \mathrm{mg} \mathrm{mL}^{-1}$. The CIN regression curve was calculated in the range $0.6-1.2 \mathrm{mg} \mathrm{mL}^{-1}$, corresponding to the range $60-120 \%$ with respect to the test concentration of $1 \mathrm{mg} \mathrm{mL}^{-1}$. The regression curves for the impurities were from the respective limit of quantitation (LOQ) to $1 \%$ with respect to the test concentration of the main compound: $\mathrm{I}_{1}, 0.0005-0.0100 \mathrm{mg} \mathrm{mL}^{-1} ; \mathrm{I}_{2}, 0.0010-0.0100 \mathrm{mg} \mathrm{mL}^{-1}$; S-CIN 0.0010-0.0100 $\mathrm{mg} \mathrm{mL}^{-1}$.

MODDE 10 software [44] was used for generating the BoxBehnken design used for the RSM, to perform the related data treatment and to draw the risk of failure maps by Monte-Carlo simulations. Nemrod-W software [45] was used for generating the Plackett-Burman design used for robustness testing. The runs of the experimental plans were carried out in a randomized order with a 
test solution containing $1 \mathrm{mg} \mathrm{mL}^{-1} \mathrm{CIN}$ and $0.0100 \mathrm{mg} \mathrm{mL}^{-1} \mathrm{CIN}$ impurities ( $1 \%$ with respect to the main compound).

\section{Results and discussion}

The analytical target profile of the method was defined as the accurate simultaneous determination of the main compound CIN and its impurities, including the chiral impurity S-CIN, in a short analysis time. The final outcome of the method should be the ability to determine the impurities at a concentration equal or lower than $0.1 \%$ with respect to $\mathrm{CIN}$, in order to find its application in the routine quality control of pharmaceutical dosage forms.

\subsection{Method scouting and critical method attributes}

Prior knowledge is the fundamental keystone of analytical development strategies, as it can effectively address the preliminary experiments in order to approach the analytical target. In this context, preliminary experiments of the scouting phase were carried out to select the separation operative mode and to identify a suitable chiral selector in order to reach an adequate selectivity. In these experiments, the concentration value for CIN did not correspond to the final test concentration value $\left(1 \mathrm{mg} \mathrm{mL}^{-1}\right)$, but it was kept low as $0.004 \mathrm{mg} \mathrm{mL}^{-1}$, as well as for S-CIN, in order to obtain clear indications on the resulting electrophoretic pattern and thus on the capability of the tested chiral selectors to achieve enantioresolution.

CIN and its impurities are $\mathrm{pH}$ sensitive compounds with an amino group in their structure $\left(\mathrm{CIN} \mathrm{pK}_{\mathrm{a}}=8.4\right)$, thus possessing basic properties and presenting a positive electrophoretic mobility at acidic $\mathrm{pH}$ values. Hence, a set of acidic conditions was evaluated using as BGE different types of buffer at different concentration values in the range $10-150 \mathrm{mM}$ and $\mathrm{pH}$ values in the range 2.50-3.50: phosphate, phosphate/acetate, acetate/borate and Britton-Robinson buffers. In order to achieve enantioseparation, several types of CyDs, mentioned in Section 2.1 , were evaluated as chiral selectors at two concentration levels $(10-30 \mathrm{mM})$. The best results in terms of analysis time and selectivity were obtained using $150 \mathrm{mM}$ phosphate buffer, with only CE $\beta C y D$ and HP $\gamma$ CyD leading to the enantioseparation among the tested CyDs. Considering that the chosen operative mode should be able to separate the enantiomers also at the test concentration value of the compounds, it was evaluated if the use of a double cyclodextrin system could improve the separation, using CE $\beta C y D$ and $\mathrm{HP} \gamma \mathrm{CyD}$ at different combinations of concentration values, ranging from 1.5 to $10.0 \mathrm{mM}$. The observed effect on the electrophoretic pattern was some improvement in enantioseparation together with a significant increase in analysis time, as shown in the representative electropherograms reported in Supplementary Fig. S1. Anyway, the increase in enantioseparation was not deemed as sufficient for selecting this separation system, considering that a favorable balance between resolution and analysis time should be achieved.

The selection between CE $\beta C y D$ and HP $\gamma C y D$ was based on the finding that they caused an opposite enantiomer migration order, being $\mathrm{R} / \mathrm{S}$ and $\mathrm{S} / \mathrm{R}$ when using $\mathrm{CE} \beta \mathrm{CyD}$ and $\mathrm{HP} \gamma \mathrm{CyD}$, respectively. As a matter of facts, adding HP $\gamma$ CyD to the BGE the main compound CIN corresponded to the last migrating peak, thus allowing the detection and determination of S-CIN avoiding any interference due to a possible peak tailing of the main drug. The addition of organic solvent modifiers ( $\mathrm{MeOH}$, acetonitrile, $n$-butanol and urea) was evaluated in the range $5-10 \% \mathrm{v} / \mathrm{v}$, showing the best results in terms of selectivity when $\mathrm{MeOH}$ was added to the BGE.

Taking into account the obtained results, the selected operative mode was capillary zone electrophoresis using as plain buffer
$150 \mathrm{mM}$ phosphate with the addition of HP $\gamma C y D$ as chiral selector and methanol as organic modifier. In these conditions the migration order of the analytes was: MET (internal standard), $\mathrm{I}_{1}, \mathrm{I}_{2}, S$-CIN and CIN. Resolution $R s_{1}$ between MET and $\mathrm{I}_{1}$, resolution $R s_{2}$ between $\mathrm{I}_{1}$ and $\mathrm{I}_{2}$ and resolution $R s_{3}$ between $\mathrm{I}_{2} / S$-CIN did not represent critical analytical issues, hence the CMAs were selected as the resolution $R s_{4}$ between $S$-CIN and CIN enantiomers and analysis time $(t)$. The CMA requirements were chosen as $R s_{4} \geq 0.5$, corresponding to a baseline separation taking into account the different concentration of the main compound and the $S$-enantiomer, and $t \leq 11 \mathrm{~min}$, in order to obtain the separation in a reasonable time.

\subsection{Risk assessment and critical method parameters}

In this study, the Ishikawa fishbone diagram [46] shown in Fig. 2 was used to formalize the risk assessment and to point out the risk factors associated with the performances of the CE analysis. The different sources of factors were represented by injection, separation/detection, capillary and BGE. The CNX tool [47] was employed for further classifying the Ishikawa parameters in parameters which should be controlled (C), potential noise parameters $(\mathrm{N})$ and parameters which should be experimented to determine acceptable ranges $(\mathrm{X})$. The results of the experiments of the scouting phase made it possible to fix injection, detection, capillary factors and BGE factors such as type and concentration of buffer, type of organic modifier, type of CyD and its degree of substitution. Instead, voltage among the separation factors and other BGE characteristics needed to be further risk managed and in-depth studied by experimental design in order to enhance the knowledge of their effects on the CMAs.

The fixed parameters were $150 \mathrm{mM}$ phosphate buffer, $18{ }^{\circ} \mathrm{C}$ capillary temperature, $3 \mathrm{~s}$ injection time at $50 \mathrm{mbar}, 48.5 \mathrm{~cm}$ capillary length and detection wavelength at $220 \mathrm{~nm}$. The selected CMPs for the subsequent multivariate study were represented by voltage $(V)$, buffer $\mathrm{pH}(p H)$, HP $\gamma C y D$ concentration $(C y D c o n c)$ and $\mathrm{MeOH}$ concentration (MeOH conc).

\subsection{Response surface methodology and method operable design region}

RSM was employed for achieving a predictive model which adequately represents the variation of the CMAs inside the selected experimental domain of the four CMPs, which is reported in Supplementary Table S1. The selected range for $p H$ and $C y D$ conc was rather narrow, according to the experimental findings from the scouting phase which clearly indicated the advantages of keeping low levels of $\mathrm{pH}$ and low levels of $C y D$ conc for obtaining a reasonable analysis time. A Box-Behnken design was selected for estimating the second order polynomial equation relating the CMPs and the CMAs and including linear, quadratic and interaction effects:

$$
\begin{aligned}
\mathrm{y} & =\beta_{0}+\beta_{1} \mathrm{x}_{1}+\beta_{2} \mathrm{x}_{2}+\beta_{3} \mathrm{x}_{3}+\beta_{4} \mathrm{x}_{4}+\beta_{11} \mathrm{x}_{1}{ }^{2}+\beta_{22} \mathrm{x}_{2}{ }^{2}+\beta_{33} \mathrm{x}_{3}{ }^{2} \\
& +\beta_{44} \mathrm{x}_{4}{ }^{2}+\beta_{12} \mathrm{x}_{1} \mathrm{x}_{2}+\beta_{13} \mathrm{x}_{1} \mathrm{x}_{3}+\beta_{14} \mathrm{x}_{1} \mathrm{x}_{4}+\beta_{23} \mathrm{x}_{2} \mathrm{x}_{3}+\beta_{24} \mathrm{x}_{2} \mathrm{x}_{4} \\
& +\beta_{34} \mathrm{x}_{3} \mathrm{x}_{4}+\varepsilon
\end{aligned}
$$

where $y$ represents the experimental response, $x_{i}$ the independent evaluated factors, $\beta_{0}$ the intercept, $\beta_{i}$ the true coefficients and $\varepsilon$ the experimental error.

Table 1 shows the 27-runs experimental plan and the measured responses including three center points for estimating the experimental variance. In this three-level design, the $k$ variables are varied two at a time by $2^{2}$ designs, while maintaining the remaining ( $k$ 2 ) variables fixed at their middle level, and it is a very efficient design in terms of required runs [41]. The responses $R s_{4}$ and $t$ were reverse transformed $\left(\mathrm{Y}^{-1}\right)$ and some of the coefficients without 


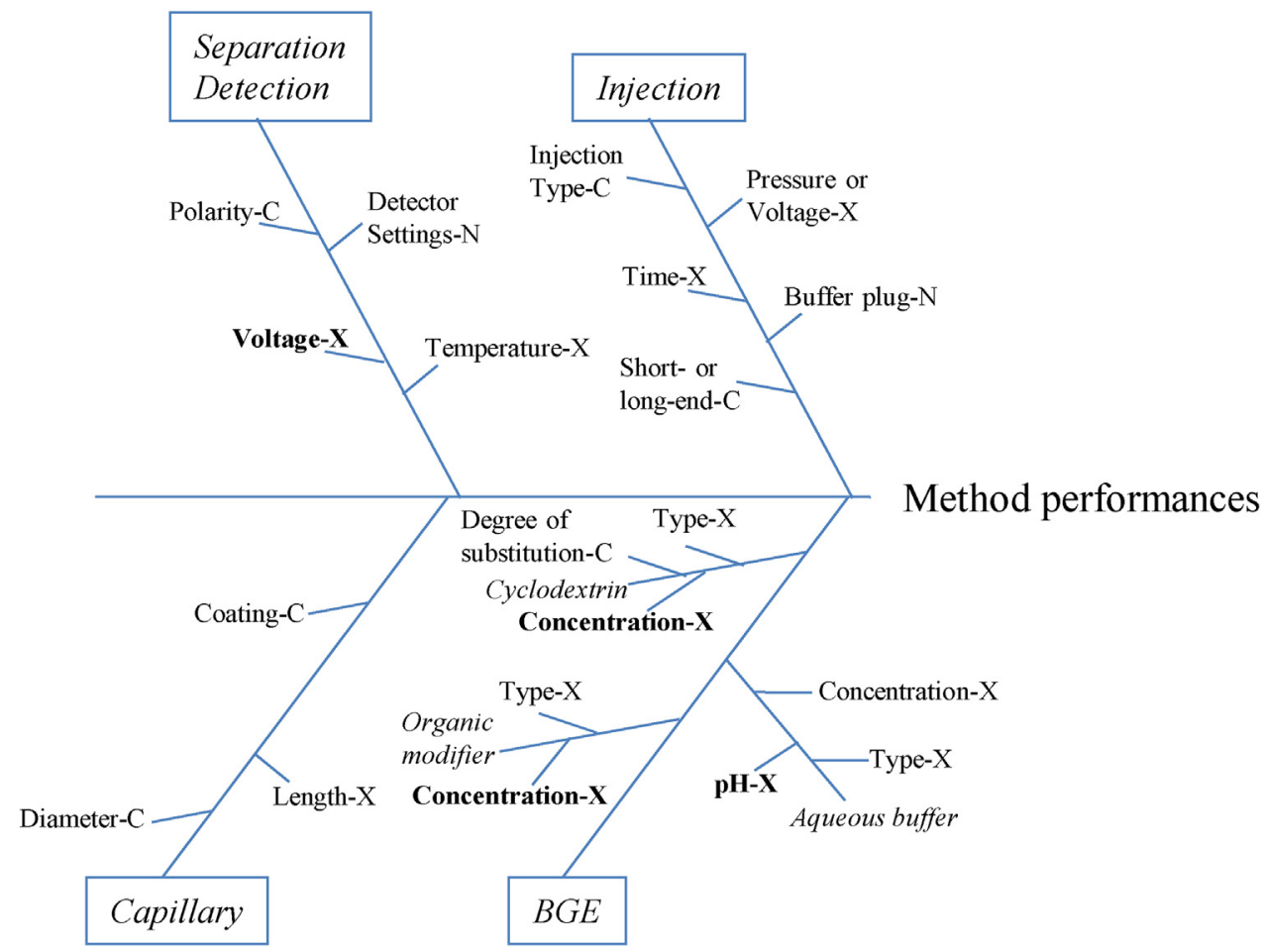

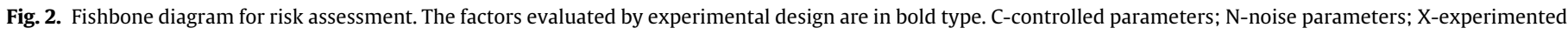
parameters.

Table 1

Response surface methodology: Box-Behnken design.

\begin{tabular}{|c|c|c|c|c|c|c|}
\hline Exp. no. & $p H$ & $\begin{array}{l}C y D \\
\text { conc } \\
(\mathrm{mM})\end{array}$ & $\begin{array}{l}\mathrm{MeOH} \\
\text { conc } \\
(\% \mathrm{v} / \mathrm{v})\end{array}$ & $\begin{array}{l}V \\
(\mathrm{kV})\end{array}$ & $R s_{4}$ & $\begin{array}{l}t \\
(\min )\end{array}$ \\
\hline 1 & 2.9 & 1.5 & 5.00 & 27 & 0.35 & 8.63 \\
\hline 2 & 2.5 & 3.5 & 5.00 & 27 & 0.47 & 9.00 \\
\hline 3 & 2.9 & 3.5 & 5.00 & 27 & 0.60 & 10.13 \\
\hline 4 & 2.7 & 2.5 & 0.00 & 24 & 0.47 & 10.53 \\
\hline 5 & 2.7 & 2.5 & 10.00 & 24 & 0.67 & 11.24 \\
\hline 6 & 2.7 & 2.5 & 0.00 & 30 & 0.55 & 8.85 \\
\hline 7 & 2.7 & 2.5 & 10.00 & 30 & 0.47 & 6.56 \\
\hline 8 & 2.5 & 2.5 & 5.00 & 24 & 0.60 & 8.20 \\
\hline 9 & 2.9 & 2.5 & 5.00 & 24 & 0.58 & 11.20 \\
\hline 10 & 2.5 & 2.5 & 5.00 & 30 & 0.48 & 12.20 \\
\hline 11 & 2.9 & 2.5 & 5.00 & 30 & 0.34 & 7.24 \\
\hline 12 & 2.7 & 1.5 & 0.00 & 27 & 0.42 & 8.03 \\
\hline 13 & 2.7 & 3.5 & 0.00 & 27 & 0.45 & 7.70 \\
\hline 14 & 2.7 & 1.5 & 10.00 & 27 & 0.90 & 9.20 \\
\hline 15 & 2.7 & 3.5 & 10.00 & 27 & 0.48 & 9.07 \\
\hline 16 & 2.5 & 2.5 & 0.00 & 27 & 0.83 & 10.74 \\
\hline 17 & 2.9 & 2.5 & 0.00 & 27 & 0.60 & 8.70 \\
\hline 18 & 2.5 & 2.5 & 10.00 & 27 & 0.50 & 9.34 \\
\hline 19 & 2.9 & 2.5 & 10.00 & 27 & 0.56 & 10.37 \\
\hline 20 & 2.7 & 1.5 & 5.00 & 24 & 0.53 & 10.31 \\
\hline 21 & 2.7 & 3.5 & 5.00 & 24 & 0.62 & 10.92 \\
\hline 22 & 2.7 & 1.5 & 5.00 & 30 & 0.70 & 12.90 \\
\hline 23 & 2.7 & 3.5 & 5.00 & 30 & 0.40 & 6.50 \\
\hline 24 & 2.7 & 2.5 & 5.00 & 27 & 0.61 & 7.74 \\
\hline 25 & 2.7 & 2.5 & 5.00 & 27 & 0.46 & 9.56 \\
\hline 26 & 2.7 & 2.5 & 5.00 & 27 & 0.43 & 9.55 \\
\hline 27 & 2.9 & 1.5 & 5.00 & 27 & 0.42 & 9.53 \\
\hline
\end{tabular}

$R s_{4}$, resolution between $S$-CIN and CIN; $t$, analysis time.

significant effect among the interaction and quadratic terms were removed in order to ameliorate the goodness of fitting (coefficient of determination $\mathrm{R}^{2}$ ) and prediction (coefficient of goodness of prediction $\mathrm{Q}^{2}$ ) of the models, calculated by multiple linear regression. In terms of ANOVA the refined model for $R s_{4}$ was both valid and significant, while a lack of fit was evidenced for $t$ model. For this response the lack of validity could be explained by the extremely high value of reproducibility observed, which refers to the pure error compared to the total variation of the response. As a consequence, the very low experimental variance of the response $t$ is not sufficient to justify the deviations of the measured responses from the model. However, all the other values of performance indicators obtained for both the models after model refining were very good [48], and the models could be employed for the prediction of the CMAs values throughout the experimental domain: $R s_{4}, \mathrm{Q}^{2}=0.569, \mathrm{R}^{2}=0.897$, reproducibility $=0.94214 ; t, \mathrm{Q}^{2}=0.831$, $\mathrm{R}^{2}=0.949$, reproducibility $=0.99993$.

The contour plots are shown in Fig. 3 and were drawn by plotting $\mathrm{CyD}$ conc vs. $\mathrm{pH}$ at three different values of $\mathrm{MeOH}$ conc $(0.0$, $5.0,10.0 \% \mathrm{v} / \mathrm{v})$, maintaining the voltage at its middle value $(27 \mathrm{kV})$. It can be observed that for $R s_{4}$ (Fig. 3a) the higher predicted values were obtained in the zone at low values of $\mathrm{pH}$ and high values of $C \gamma D$ conc, both at low values and at high values of $\mathrm{MeOH}$ conc, pointing out the presence of a quadratic effect of the latter factor. As for $t$ (Fig. 3b), the lower predicted values were observed at a low level of $\mathrm{MeOH}$ conc, of $\mathrm{CyD}$ conc and of $\mathrm{pH}$. The curvatures of the isoresponse lines of the contour plots were further clarified by the direct analysis of the coefficients, shown in Supplementary Fig. $\mathrm{S} 2$. The graphic analysis of effects confirmed that $\mathrm{MeOH}$ conc presented a significant quadratic effect on $R s_{4}$, as already evidenced by the analysis of the related contour plots. Moreover, a quadratic effect of $C y D$ conc and interaction effects between $V$ and $p H$ and between $p H$ and $C y D$ conc were highlighted, with opposite sign. As for $t$, an important quadratic effect was shown for $V$, along with a relevant interaction between $\mathrm{V}$ and $\mathrm{MeOH}$ conc. The quadratic effect of $\mathrm{MeOH}$ conc on $\mathrm{Rs}_{4}$ may be explained hypothesizing that at low concentration levels (i.e., within 5\%), the addition of the organic solvent led to the enantioresolution decrease as a result of the diminished affinity of the analyte for the CyD. In particular, the observed situation, related to a decrease of selectivity, typically occurs when the concentration of the CyD is at or below the optimum value for the organic solvent free buffer [49]. Interestingly, at 
(a) $R s_{4}$

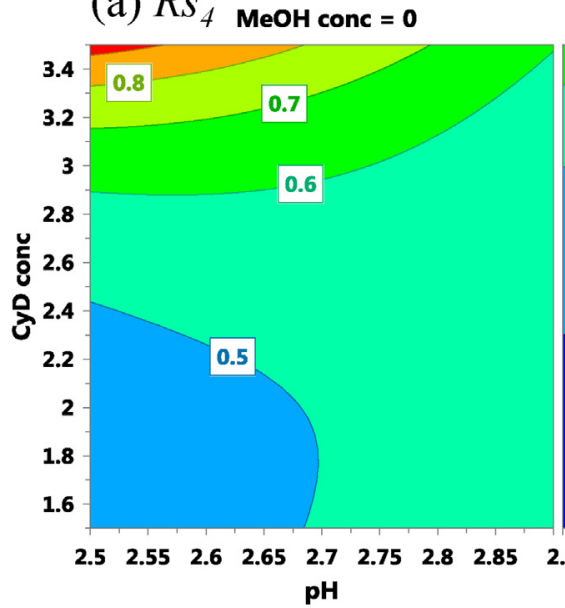

(b) $t$

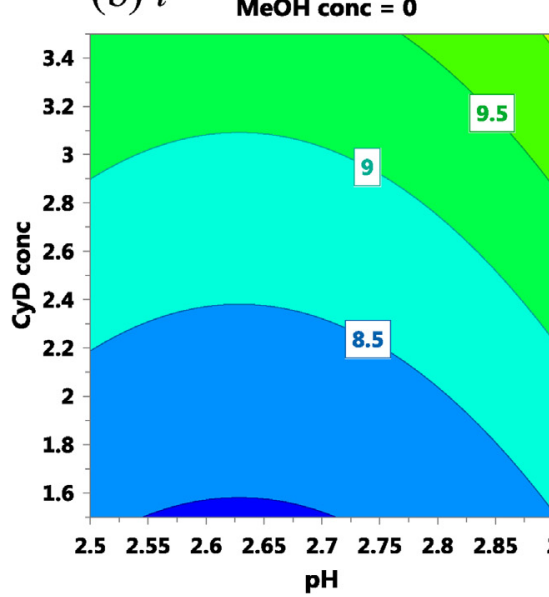

MeOH conc $=\mathbf{5}$

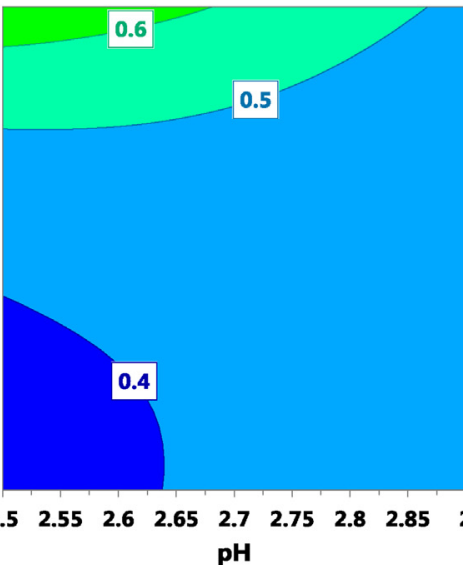

pH

MeOH conc $=\mathbf{5}$

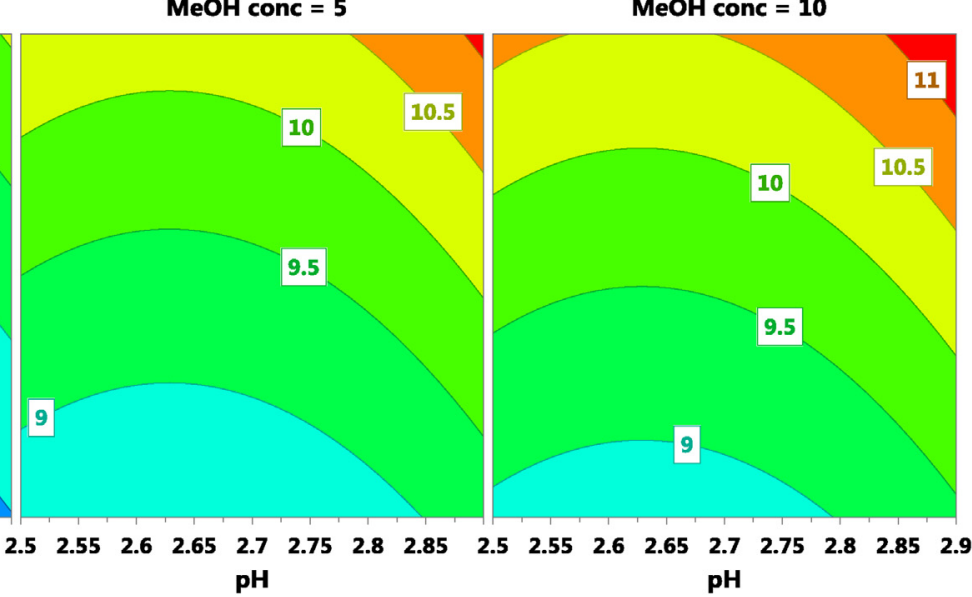

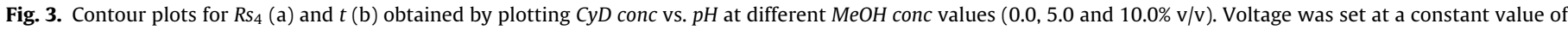
$27 \mathrm{kV}$.

higher $\mathrm{MeOH}$ concentration values (i.e., 5-10\%) the enantioresolution was found to progressively improve likely due to the effects of the organic solvent on the viscosity, dielectric constant and conductivity of the background electrolyte, which in the considered situation positively affected the separation efficiency [50,51]. This was also confirmed by performing the response surface study using as response the number of theoretical plates $N$ of CIN peak. A negative quadratic effect of $\mathrm{MeOH}$ conc on $N$ was pointed out, as shown in the related factor effect plot displayed in Supplementary Fig. S3.

Sweet spot plots were drawn in order to get the combinations of the CMP which led to accepted values of both CMAs and are shown in Fig. 4 at three different $\mathrm{MeOH}$ conc values and a constant voltage of $27 \mathrm{kV}$. The region in green corresponds to the fulfilled requirements for both CMAs, while the region where only one CMA requirement was fulfilled, without specifying which one, is depicted in blue. From the analysis of these plots, it is possible to note once again that mainly due to the strong quadratic effect of $\mathrm{MeOH}$ conc on $\mathrm{Rs}_{4}$ the green zone was much wider at low and high values of this CMP. However, the MODR cannot be simply defined as corresponding to the green zone in the sweet spot plots. For identifying the MODR, it is crucial to consider model uncertainty because this region corresponds to the set of experimental conditions where all the CMAs fulfill the requirements with a certain probability $[28,31]$. Hence, the estimation of the probability map of the present CE method was based on the calculated models and their uncertainty, performing a risk analysis by using Monte-Carlo simulations [42] on the factors' settings. The possible factor ranges were expanded symmetrically by a search function of the software MODDE [44] from a set-point to the widest possible range until one response limit was exceeded in terms of specified DPMO (defects per million opportunities). The DPMO value was set as 100,000 (10\% risk of failure), and the calculated MODR was identified in the probability map reported in Fig. 5 as the zone where the risk of error is $\leq 10 \%$ (green area), corresponding to the following ranges: $V, 26-27 \mathrm{kV}, \mathrm{pH}, 2.60-2.80$; $\mathrm{CyD}$ conc, 3.0-3.5 mM; $\mathrm{MeOH}$ conc, $0.00-8.40 \% \mathrm{v} / \mathrm{v}$. It is worthwhile to note that the limits for $p H$ enclosed a very narrow range and that the addition of $\mathrm{MeOH}$ conc could be useful for increasing $R s_{4}$ but was not strictly necessary to fulfill the desired requirements.

In order to validate the MODR some experiments were performed at the extremes of the CMP ranges and the CMAs were evaluated to verify that the requirements were fulfilled also in these extreme points. The experimental conditions were selected according to a Plackett-Burman matrix where the level +1 and -1 for each CMPs corresponded to the higher and the lower limits of the DS range, respectively. The results obtained by applying these conditions showed a good accordance between the predicted and the measured CMAs. After validation of the MODR, a working point was chosen inside the lower risk region, taking into account some practical considerations [52] such as the advantages of keeping low the generated current by using the lower value of voltage and of using a low concentration of HP $\gamma$ CyD and methanol for maintaining low 

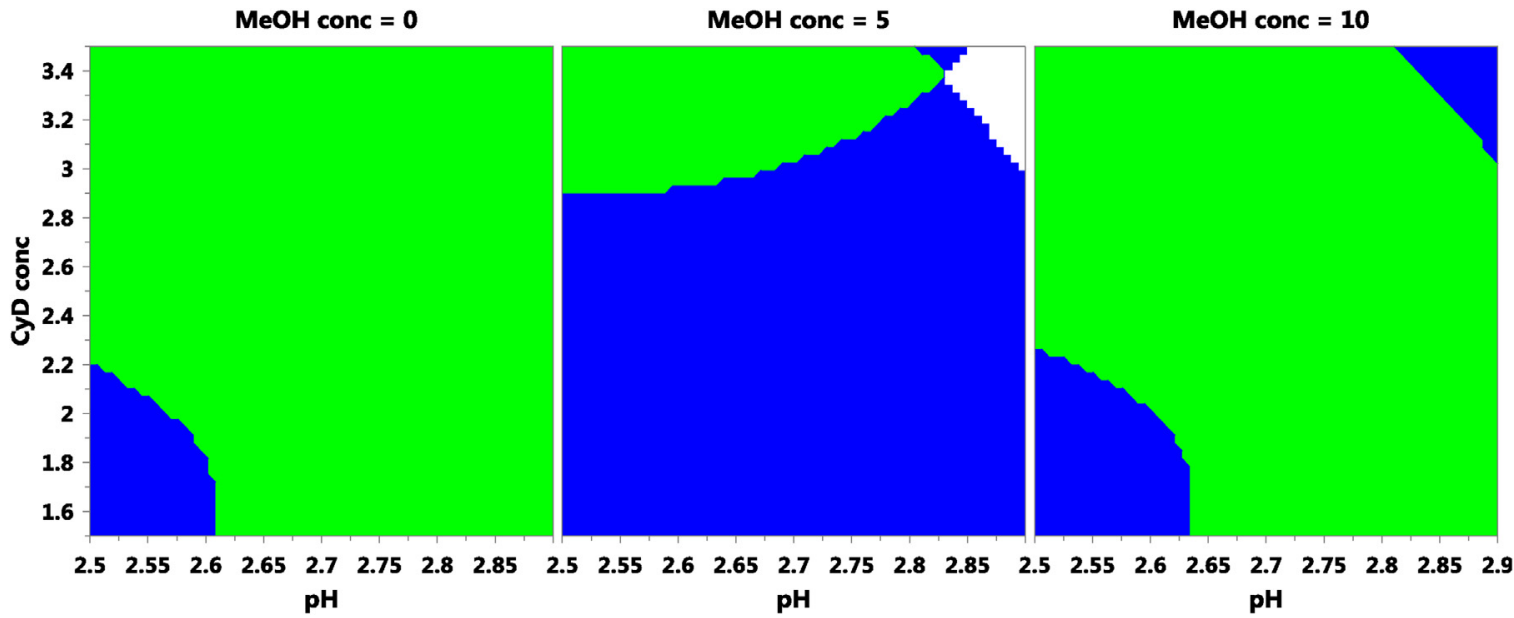

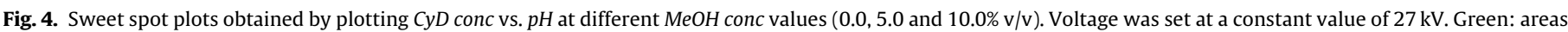

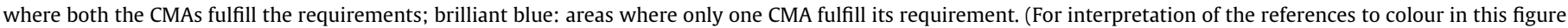
legend, the reader is referred to the web version of this article).
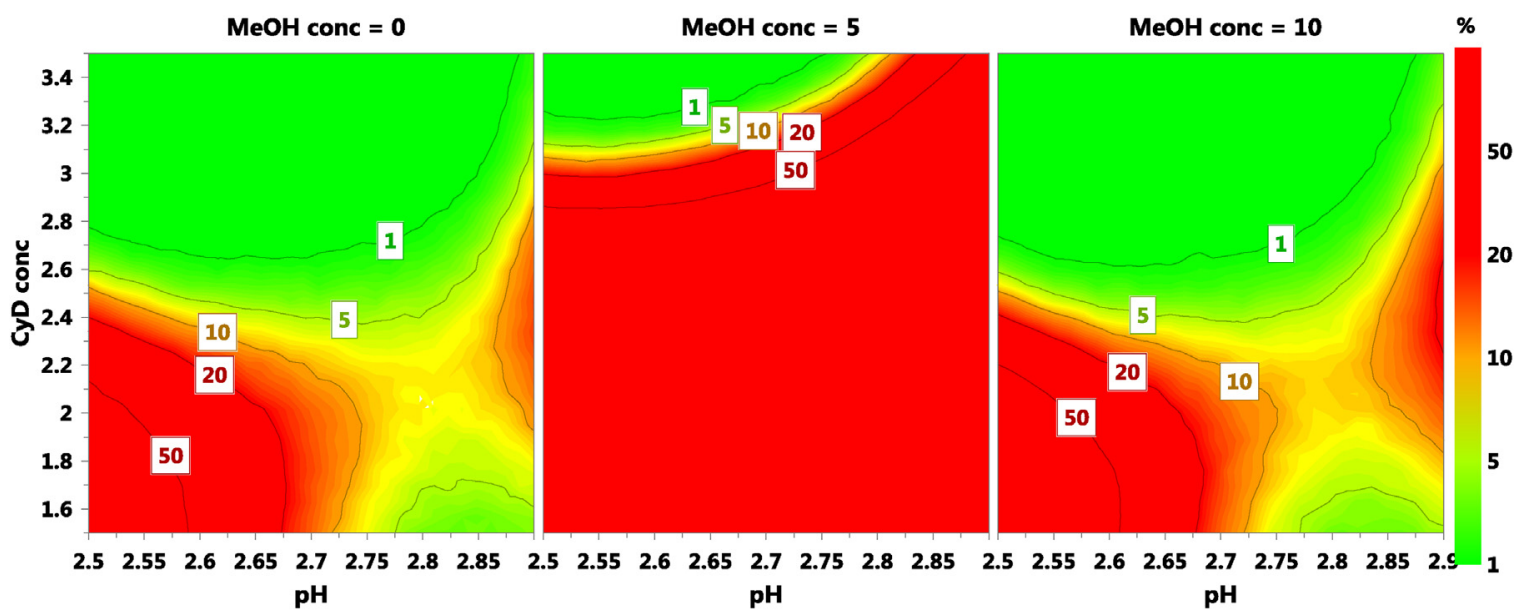

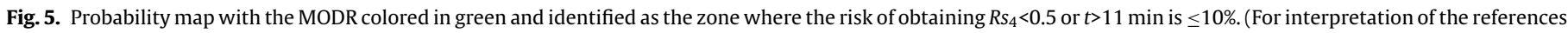
to colour in this figure legend, the reader is referred to the web version of this article).

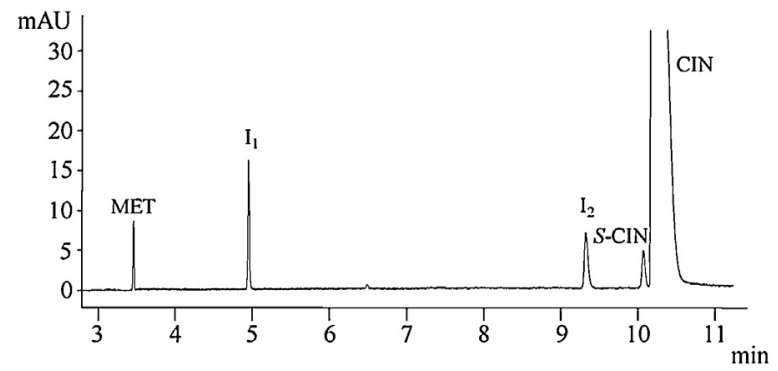

Fig. 6. Electropherogram obtained with the working points conditions. Sample: CIN, $1 \mathrm{mg} \mathrm{mL}^{-1}$; CIN impurities, $0.01 \mathrm{mg} \mathrm{mL}^{-1}$; MET, $0.06 \mathrm{mg} \mathrm{mL}^{-1}$. Detection wavelength: $220 \mathrm{~nm}$. Experimental conditions: capillary length, $48.5 \mathrm{~cm}$; temperature, $18^{\circ} \mathrm{C}$; voltage, $26 \mathrm{kV}$; BGE, $150 \mathrm{mM}$ phosphate buffer $\mathrm{pH} 2.70,3.1 \mathrm{mM}$ HP $\gamma \mathrm{CyD}, 2.00 \%$ $\mathrm{v} / \mathrm{v} \mathrm{MeOH}$.

costs of analysis. The selected working point for routine analysis was $26 \mathrm{kV}$, pH 2.70, 3.1 mM CyD conc and 2.00\% v/v MeOH conc. The electropherogram obtained when applying these conditions is reported in Fig. 6, showing a baseline separation of all the analytes in less than $11 \mathrm{~min}$, with a generated current of about $85 \mu \mathrm{A}$.

\subsection{Robustness and method control}

A multivariate approach was employed to test the method robustness, examining the effect on the CMAs of the change of all the four CMPs and of the factors temperature $(T)$ and phosphate concentration (buffer conc) in a small interval [43]. The center of the considered experimental domain corresponded to the working point conditions: $V, 25-27 \mathrm{kV} ; \mathrm{pH}, 2.60-2.80$; $C y D$ conc, 2.8-3.4 mM; $\mathrm{MeOH}$ conc, $1.80-2.20 \% \mathrm{v} / \mathrm{v} ; \mathrm{T}, 17-19^{\circ} \mathrm{C}$; buffer conc, 148-152 mM. A linear model was postulated for relating the factors and the CMAs in the small interval considered and a PlackettBurman design was employed for estimating the coefficients. The experimental plan is reported in Supplementary Table S2 with the measured responses. Both the models resulted not significant, evidencing that the variation of the factors in the interval considered did not lead to a significant variation in the responses. From these results, method control was accomplished by selecting as system suitability limits the lower and the higher values for the CMAs observed during robustness testing: $0.53<R s_{4}<0.71,9.43<t<11.20$.

\subsection{Validation and application}

Validation of the method was carried out in compliance to ICH guideline Q2(R1) [43]. The validation data are reported in the 
Supplementary Content, showing adequate performances for the intended use. The developed method was finally applied for the analysis of a real sample of Mimpara ${ }^{\circledR}$ tablets containing $90 \mathrm{mg}$ CIN and a typical electropherogram is shown in Supplementary Fig. S4a. Four analyses were performed and the results were in agreement with the declared content $(\alpha / 2=0.025)$ : percentage of label claim, $98.6 \pm 1.5 \%$; RSD, $1.0 \%$. None of the three considered impurities was detected. Supplementary Fig. S4b shows the analysis of the real sample spiked with the impurities at the LOQ concentration values.

\section{Conclusions}

In drug analysis, the simultaneous determination of enantiomeric purity and impurity assay represents a challenge to the analytical technique for the separation performances required in consequence of the necessary overloading of the main enantiomer and of the similarity of the chemical structures of the compounds. To deal with these analytical issues, a systematic strategy such as QbD could facilitate method development and should be preferably followed for its advantages in terms of gained knowledge and risk management. With this aim, in this study a CE method for simultaneously performing enantiomeric purity control and impurity profiling of CIN was effectively developed by applying QbD methodology. A high degree of analytical method understanding was acquired by the application of multivariate tools, highlighting the presence of interaction and quadratic effects of the considered CMPs on both enantioresolution and analysis time. The final outcome of QbD framework was the definition of the MODR, a multivariate zone of input parameters where the desired performances of the method were obtained with a selected degree of probability $\pi \geq 90 \%$. Implementation of $\mathrm{QbD}$ was demonstrated to provide a practical and effective roadmap leading to the analytical target profile. After validation, the developed method was satisfactorily applied to the analysis of a CIN pharmaceutical product.

\section{Acknowledgements}

The Authors wish to thank Zentiva, k.s. Praha, a Sanofi Company (Czech Republic) for the kind gift of reference standards of CIN and its impurities, and Ente Cassa di Risparmio di Firenze, Italy (Contributo n. 2014.0577) and Estancias de movilidad en el extranjero "José Castillejo" of the Spanish "Ministerio de Educación, Cultura y Deporte" (CAS17/00140) for the financial support.

\section{Appendix A. Supplementary data}

Supplementary material related to this article can be found, in the online version, at doi:https://doi.org/10.1016/j.chroma.2018. 07.021.

\section{References}

[1] H. Liu, H. Wang, T. Liu, J. Jiang, X. Chen, F. Gao, P. Hu, Pharmacokinetic and pharmacodynamic properties of cinacalcet (KRN1493) in Chinese healthy volunteers: a randomized, open-label, single ascending-dose and multiple-dose, parallel-group study, Clin. Ther. 38 (2016) 348-357.

[2] C.H. Wei, A. Harari, Parathyroid carcinoma: update and guidelines for management, Curr. Treat. Options Oncol. 13 (2012) 11-23.

[3] E.F. Nemeth, W.H. Heaton, M. Miller, J. Fox, M.F. Balandrin, B.C. Van Wagenen, M. Colloton, W. Karbon, J. Scherrer, E. Shatzen, G. Rishton, S. Scully, M. Qi, R. Harris, D. Lacey, D. Martin, Pharmacodynamics of the type II calcimimetic compound cinacalcet HCl, J. Pharmacol. Exp. Ther. 308 (2004) 627-635.

[4] D.C. Patel, M.F. Wahab, D.W. Armstrong, Z.S. Breitbach, Advances in high-throughput and high-efficiency chiral liquid chromatographic separations, J. Chromatogr. A 1467 (2016) 2-18.

[5] M. Catani, O.H. Ismail, F. Gasparrini, M. Antonelli, L. Pasti, N. Marchetti, S Felletti, A. Cavazzini, Recent advancements and future directions of superficially porous chiral stationary phases for ultrafast high-performance enantioseparations, Analyst 142 (2017) 555-566.
[6] S. Fanali, Nano-liquid chromatography applied to enantiomers separation, J. Chromatogr. A 1486 (2017) 20-34.

[7] M. Del Bubba, L. Checchini, L. Lepri, Thin-layer chromatography enantioseparations on chiral stationary phases: a review, Anal. Bioanal. Chem. 405 (2013) 533-554

[8] Q. Zhu, G.K.E. Scriba, Analysis of small molecule drugs, excipients and counter ions in pharmaceuticals by capillary electromigration methods - recent developments, J. Pharm. Biomed. Anal. 147 (2018) 425-438.

[9] S. El Deeb, H. Wätzig, D.A. El-Hady, C. Sänger-van de Griend, G.K.E. Scriba, Recent advances in capillary electrophoretic migration techniques for pharmaceutical analysis (2013-2015), Electrophoresis 37 (2016) 1591-1608.

[10] B. Chankvetadze, Combined approach using capillary electrophoresis and NMR spectroscopy for an understanding of enantioselective recognition mechanisms by cyclodextrins, Chem. Soc. Rev. 33 (2004) 337-347.

[11] A. Servais, A. Rousseau, M. Fillet, K. Lomsadze, A. Salgado, J. Crommen, B. Chankvetadze, Separation of propanolol enantiomers by CE using sulfated $\beta-C D$ derivatives in aqueous and non-aqueous electrolytes: comparative CE and NMR study, Electrophoresis 31 (2010) 1467-1474

[12] F. Melani, B. Pasquini, C. Caprini, R. Gotti, S. Orlandini, S. Furlanetto, Combination of capillary electrophoresis, molecular modeling and NMR to study the enantioselective complexation of sulpiride with double cyclodextrin systems, J. Pharm. Biomed. Anal. 114 (2015) 265-271.

[13] A. Salgado, B. Chankvetadze, Applications of nuclear magnetic resonance spectroscopy for the understanding of enantiomer separation mechanisms in capillary electrophoresis, J. Chromatogr. A 1467 (2016) 95-144.

[14] G. D’Orazio, C. Fanali, M. Asensio-Ramos, S. Fanali, Chiral separations in food analysis, Trends Anal. Chem. 96 (2017) 151-171.

[15] I.M. Mavidris, K. Yannakopoulou, Anionic cyclodextrins as versatile hosts for pharmaceutical nanotechnology: synthesis, drug delivery, enantioselectivity, contrast agents for MRI, Int. J. Pharm. 492 (2015) 275-290.

[16] B. Pasquini, F. Melani, C. Caprini, M. Del Bubba, S. Pinzauti, S. Orlandini, S. Furlanetto, Combined approach using capillary electrophoresis, NMR and molecular modeling for ambrisentan related substances analysis: investigation of intermolecular affinities, complexation and separation mechanism, J. Pharm. Biomed. Anal. 144 (2017) 220-229.

[17] A. Sigala, C.H.V. Raghunath Babu, M. Satish Varma, G. Balaswamy, A new validated liquid chromatographic method for the determination of impurities in cinacalcet hydrochloride, Anal. Chem. Ind. J. 8 (2009) 594-599.

[18] M. Krishnan, S.L. Karunanidhi, G. Sola, Y. Akshitha, Stability indicating HPLC method for the estimation of cinacalcet hydrochloride API, Indian J. Res. Pharm. Biotechnol. 1 (2013) 346-350.

[19] E. Rao Bammidi, V. Lakinani, D.K.G. Levi, Method development and validation and degradation studies for cinacalcet $\mathrm{HCl}$ drug by RP-HPLC method, Int. J. Chrom. Pharm. Sci. 2 (2014) 990-996.

[20] R. Nageswara Rao, S. Saida, B. Sravan, B. Ramesh, Liquid chromatographic separation, determination and ESI-MS/MS, FT-IR and NMR characterization of the forced degradation products of cinacalcet, Anal. Methods 6 (2014) 5076-5087

[21] P. Sunil Reddy, T.V.R. Raju, P.S. Raju, N.S. Varma, K.S. Babu, Development and validation of a stability-indicating RP-UPLC method for the estimation of impurities in cinacalcet hydrochloride API and its formulation, Sci. Pharm. 83 (2015) 583-598

[22] V. Ravinder, S. Ashok, M. Satish Varma, C.V. Raghunath Babu, K. Shanker, G. Balaswamy, A validated chiral LC method for the enantiomeric separation of cinacalcet hydrochloride, Chromatographia 70 (2009) 229-232.

[23] R. Bhushan, R. Dubey, Indirect reversed-phase high-performance liquid chromatographic and direct thin-layer chromatographic enantioresolution of (R,S)-Cinacalcet, Biomed. Chromatogr. 25 (2011) 674-679.

[24] M. Douša, J. Břicháč, Chiral chromatography studies of chemical behavior of cinacalcet on polysaccharide chiral reversed-phase HPLC stationary phases, J. AOAC Int. 95 (2012) 1639-1645

[25] R. Nageswara Rao, S. Bompelli, LC-MS/MS determination of cinacalcet enantiomers in rat plasma on Chirobiotic V column in polar ionic mode: application to a pharmacokinetic study, Biomed. Chromatogr. 28 (2014) 1846-1853

[26] P. Ginterová, J. Znaleziona, R. Knob, M. Douša, J. Petr, J. Ševčik, Enantiomeric purity control of $R$-cinacalcet in pharmaceutical product by capillary electrophoresis, Chem. Pap. 70 (2016) 1024-1030.

[27] ICH Harmonised, Tripartite Guideline, Pharmaceutical development Q8(R2), in: International Conference on Harmonisation of Technical Requirements for Registration of Pharmaceuticals for Human Use, 2009.

[28] E. Rozet, P. Lebrun, P. Hubert, B. Debrus, B. Boulanger, Design spaces for analytical methods, Trends Anal. Chem. 42 (2013) 157-167.

[29] S. Orlandini, S. Pinzauti, S. Furlanetto, Application of quality by design to the development of analytical separation methods, Anal. Bioanal. Chem. 405 (2013) 443-450.

[30] Guidance for Industry, PAT - a framework for innovative pharmaceutical development, in: Manufacturing, and Quality Assurance, U.S Food and Drug Administration, 2004.

[31] A. Dispas, H.T. Avohou, P. Lebrun, P. Hubert, C. Hubert, "Quality by Design" approach for the analysis of impurities in pharmaceutical drug product and drug substances, Trends Anal. Chem. 101 (2018) 24-33.

[32] A.H. Schmidt, I. Molnár, Using an innovative Quality-by-Design approach for the development of a stability indicating UHPLC method for ebastine in the API and pharmaceutical formulations, J. Pharm. Biomed. Anal. 78-79 (2013) 65-74.

Please cite this article in press as: B. Pasquini, et al., Chiral capillary zone electrophoresis in enantioseparation and analysis of cinacalcet impurities: Use of Quality by Design principles in method development, J. Chromatogr. A (2018), https://doi.org/10.1016/j.chroma.2018.07.021 
[33] C. Boussès, L. Ferey, E. Vedrines, K. Gaudin, Using an innovative combination of quality-by-design and green analytical chemistry approaches for the development of a stability indicating UHPLC method in pharmaceutical products, J. Pharm. Biomed. Anal. 115 (2015) 114-122.

[34] J. Terzić, I. Popović, A. Stajić, A. Tumpa, B. Jančić-Stojanović, Application of Analytical Quality by Design concept for bilastine and degradation impurities determination by hydrophilic interaction liquid chromatographic method, J. Pharm. Biomed. Anal. 125 (2016) 385-393.

[35] B. Andri, P. Lebrun, A. Dispas, R. Klinkenberg, B. Streel, E. Ziemons, R.D. Marini, Ph. Hubert, Optimization and validation of a fast supercritical fluid chromatography method for the quantitative determination of vitamin D3 and its related impurities, J. Chromatogr. A 1491 (2017) 171-181.

[36] S. Orlandini, B. Pasquini, M. Stocchero, S. Pinzauti, S. Furlanetto, An integrated quality by design and mixture-process variable approach in the development of a capillary electrophoresis method for the analysis of almotriptan and its impurities, J. Chromatogr. A 1339 (2014) 200-209.

[37] S. Orlandini, B. Pasquini, C. Caprini, M. Del Bubba, L. Squarcialupi, V. Colotta, S. Furlanetto, A comprehensive strategy in the development of a cyclodextrin-modified microemulsion electrokinetic chromatographic method for the assay of diclofenac and its impurities: mixture-process variable experiments and quality by design, J. Chromatogr. A 1466 (2016) 189-198.

[38] B. Pasquini, S. Orlandini, C. Caprini, M. Del Bubba, M. Innocenti, G. Brusotti, S. Furlanetto, Cyclodextrin- and solvent-modified micellar electrokinetic chromatography for the determination of captopril, hydrochlorothiazide and their impurities: a Quality by Design approach, Talanta 160 (2016) 332-339.

[39] S. Orlandini, B. Pasquini, M. Del Bubba, S. Pinzauti, S. Furlanetto, Quality by design in the chiral separation strategy for the determination of enantiomeric impurities: development of a capillary electrophoresis method based on dual cyclodextrin systems for the analysis of levosulpiride, J. Chromatogr. A 1380 (2015) 177-185.

[40] S. Orlandini, B. Pasquini, C. Caprini, M. Del Bubba, M. Douša, S. Pinzauti, S. Furlanetto, Enantioseparation and impurity determination of ambrisentan using cyclodextrin-modified micellar electrokinetic chromatography: visualizing the design space within quality by design framework, J. Chromatogr. A 1467 (2016) 363-371.
[41] G.A. Lewis, D. Mathieu, R. Phan-Tan-Luu, Pharmaceutical Experimental Design, Marcel Dekker, New York, 1999.

[42] M.A. Herrador, A.G. Asuero, A.G. Gonzalez, Estimation of the uncertainty of indirect measurements from the propagation of distributions by using the Monte-Carlo method: an overview, Chemom. Intell. Lab. Syst. 79 (2005) $115-122$.

[43] ICH Harmonised, Tripartite Guideline, Validation of analytical procedures: text and methodology Q2(R1), in: International Conference on Harmonisation of Technical Requirements for Registration of Pharmaceuticals for Human Use, 2005.

[44] MODDE v. 10, MKS Umetrics AB, Sweden.

[45] D. Mathieu, J. Nony, R. Phan-Tan-Luu, NEMROD-W, LPRAI sarl, Marseille.

[46] K. Ishikawa, What Is Total Quality Control? The Japanese Way, Prentice-Hall, Englewood Cliffs, 1985.

[47] P. Borman, M. Chatfield, P. Nethercote, D. Thompson, K. Truman, The application of Quality by Design to analytical methods, Pharm. Tech. 31 (2007) 142-152.

[48] L. Eriksson, E. Johansson, N. Kettaneh-Wold, C. Wikström, S. Wold, Design of Experiments - Principles and Applications, MKS Umetrics AB, Umeå, Sweden, 2008.

[49] S.A.C. Wren, R.C. Rowe, Theoretical aspects of chiral separation in capillary electrophoresis: II. The role of organic solvent, J. Chromatogr. A 609 (1992) 363-367.

[50] A. Van Eeckhaut, M.R. Detaevernier, J. Crommen, Y. Michotte, Differential effects of organic modifiers on the enantioseparation of dimetindene maleate with carboxymethyl- $\beta$-cyclodextrin in capillary electrophoresis, J. Sep. Sci. 27 (2004) 21-27.

[51] S. Fanali, Enantioselective determination by capillary electrophoresis with cyclodextrins as chiral selectors, J. Chromatogr. A 875 (2000) 89-122.

[52] L. Nompari, S. Orlandini, B. Pasquini, C. Campa, M. Rovini, M. Del Bubba, S. Furlanetto, Quality by design approach in the development of an ultra-high-performance liquid chromatography method for Bexsero meningococcal group B vaccine, Talanta 178 (2018) 552-562. 